\title{
Effects of Stimulus Shape on Visual Evoked Potentials
}

\author{
SHERRILL J. PUR VES AND MORTON D. LOW.
}

SUMMARY: Visual evoked responses (VER) to four geometric shapes (a square, circle, el and omega) were recorded from multiple scalp locations in twelve subjects. Significant differences were found between the occipital VERs to the square and el and between the VERs to the circle and omega. Consistent differences could not be demonstrated between the responses to the square and circle or to the el and omega.

The differences between the responses were quantified by three different methods, including measurement of peak latencies and amplitudes, computation of a ratio index called $\lambda$, and the performance of discriminant functions derived by Stepwise Discriminant Analysis Program (SWDA) in classifying other single trial responses. The amplitudes (but not the latencies) of $a$ negative peak at approximately $150 \mathrm{msec}$. and a positive one at $220 \mathrm{msec}$. latency were found to be different in the pairs of shapes described. SWDA was able to separate these same pairs and classify from 63 to $68 \%$ of new trials correctly. The latency points chosen to make up the discriminant functions correlated partly with the $\overline{N 150}$ and $\overline{P 220}$ components that were found to be significantly different in amplitude.

It is suggested that the VER differences in the earlier part of the wave-form are related to the physical properties of the stimuli, including the contrast densities in the central $1.5^{\circ}$ of the visual field and/or the spatial frequencies contained in the stimuli. Differences demonstrated in later parts of the VERs may reflect differences in symbolic meaning of the stimuli.

From the Division of Neurology, Department of Medicine, University of British Columbia and Department of Diagnostic Neurophysiology, Vancouver General Hospital, Vancouver, Canada.

Reprint requests to Dr. Sherrill J. Purves, Dept. of Medicine, Vancouver General Hospital, 855 West 12th Ave. Vancouver, B.C. V5Z 1M9 Canada.

This work was supported by a Medical Research Council of Canada Fellowship to Dr. S. Purves and MRC operating grant MT-3313. This study was part of the experimental work for the doctoral dissertation of SJP.

A preliminary version of the report was presented at the Canadian Congress of Neurological Sciences in June, 1977 for the Herbert Jasper Award in clinical neurophysiology.
RÉSUMÉ: Nous avons enregistré chez 12 sujets, à partir de plusieurs sites de surface, les potentiels évoqués visuels (VER) en réponse à quatre formes géométriques (un carré, un cercle, "el" et "omega"). Des différences significatives furent constatées dans les VER occipitaux entre le carré et le "el" et entre le cercle et l'omega. Cependant des réponses constantes ne purent être observées entre les réponses au carré et cercle ou au "el" et omega.

Les différences entre les réponses furent quantifiés à l'aide de trois méthodes: mesure des latences de pointe et des amplitudes, calcul d'un rapport nommé $\lambda$, et la performance des fonctions discriminatoires obtenues par SWDA lors de la classification des réponses aux autres essais simples. Nous avons trouvé une différence dans les amplitudes (mais non les latences) du pic négatif à $150 \mathrm{msec}$. et du pic positif à 220 msec. de latence entre les paires géométriques décrites. Le SWDA pouvait séparer ces mêmes paires et les classifier correctement dans 63 a $68 \%$ des nouveaux essais. Les points de latence choisis pour ces analyses de fonctions discriminatoires correspondaient en partie aux composantes $\overline{N 150}$ et $\overline{P 220}$ qui étaient significativement différentes quant à l'amplitude.

Nous suggérons que les différences de $V E R$ dans la première partie de l'onde correspondent aux propriétés physiques des stimuli, incluant les densités contrastantes des $1.5^{\circ}$ de la partie centrale du champ visuel et/ou les fréquences spatiales contenues dans les stimuli. Les différences observées dans les parties subséquentes du $V E R$ reflètent peut-être des différences dans le sens symbolique des stimuli.

\section{INTRODUCTION}

The study of evoked potentials has become an important subject in clinical neurophysiology in recent years. These waveforms are useful indicators of the functional state of the central nervous system under a variety of conditions, and their recording entails little discomfort and no risk for the patient or subject. However, some crucial questions, notably what specific cerebral processes and structures generate these waveforms and whether the evoked responses reflect neuronal activities related to cognitive or only the physical properties of the stimulus, are still unanswered. Because of the possibility of easily varying cognitive aspects of the stimulus without dramatically changing its physical properties, a patterned visual stimulus has often been employed in studies related to these questions. However, the experimental reports of investigations using such stimuli have so far failed to provide convincing evidence that the patterned visual evoked response (VER) represents a physiological correlate of either sensory or perceptual cerebral processes.

The effects on the VER configuration of some physical parameters of patterned visual stimuli such as luminance, size of checks in a checkerboard pattern, and field size are significant and have all been well documented (Regan, 1967; Harter, 1968; Spekreijse et al., 1973). A few authors have studied the effect on the VER of the symbolic meaning (as opposed to the physical parameters) of the stimulus. John et al. (1967), in a study of VERs elicited by blank field and geometric stimuli, found that the VERs to different visual forms of equal area were different, although evoked potentials to the same visual forms of different areas were similar. They concluded from these findings that the VER waveform 
reflected the symbolic meaning and not the physical properties of the stimulus. Buchsbaum and Fedio (1969) came to similar conclusions in a separate study of VERs elicited by different patterns of dots. By contrast, Rem and Schwartz (1976), using a complex method for stimulus presentation (Julensz's anaglyphs), which made it possible to vary the image presented to the subject without varying the physical characteristics of the stimuli arriving at the retina, also studied the effect of stimulus meaning on the VER configuration. They reasoned that while the stimulus color and the perception of a three-dimensional stimulus did effect the evoked response, changes in the figural content of the stimuli did not, and therefore concluded the VER only reflects activity related to physical feature detection and not to symbolic meaning.

All of these investigations used rather simple and in some cases idiosyncratic methods of determining waveform differences between the VERs elicited by different stimuli. In the first two studies reported above, a number or index was derived from a ratio of the sum of the voltage values of the individual latency points of the responses. The third (Rem and Schwartz, 1976) used Student's t tests to determine differences between pairs of waveforms. None of these studies quantified or even described the responses in the conventional manner, i.e. through measures of the latency and amplitude of selected waveform components. While this method does have some disadvantages (Donchin, 1969), it has been used in a great majority of the studies of the effects of physical parameters on the VER. A comparison of the results of the cognitive effect and physical parameter effect studies is therefore very difficult.

Averaged evoked potential waveforms can be analyzed, quantified, and compared by many different methods, including those alluded to above and these have been reviewed in detail elsewhere (Donchin, 1969; Regan, 1972), with discussion of their advantages and disadvantages. One complex method of determining evoked response differences that is particularly advocated by Donchin is based on multivariate statistical theory called dis- criminant analysis. It utilizes the digitized values of the individual latency points of single response trials in a classification technique that uses observations obtained from members of different groups whose group membership is know to derive criteria for the classification of an observation whose group membership is doubtful. Its use is particularly advantageous in evoked response work because it takes into account the considerable correlation that exists between successive time points in the averaged evoked response.

The purposes of the study reported in this paper were first to determine whether different stimulus shapes (similar to those used by John et al., 1967) would evoke the VERs which reflected differences in cognitive rather than just the physical aspects of the stimuli; and second to determine the statistical significance on any differences found by a variety of analysis techniques, including traditional and more complex methods such as discriminant analysis, in order to precisely characterize the VERs and the differences between them.

\section{METHODS}

Subjects were 12 paid women student volunteers between the ages of 19 and 28. All of the subjects were tested at least once and some were tested repeatedly, but always on different days separated by at least a week. Subjects were told that the experiments were intended to record EEG activity related to a variety of visual patterns. They sat alone in a shielded room with low intensity overhead lighting $(.5 \mathrm{ft}$. candles) and were instructed to visually fixate on a point on a television screen (1 meter distant) during the presentation of the different stimulus shapes and to try to stay alert and attentive.

The stimuli were the four geometric shapes shown in Fig. 1. They included two familiar shapes, a square, and a circle, and two unfamiliar and less easily named shapes called here the el and the omega. There were all shown as white outlines on a black background. All had approximately equal length of contrasting border. They were presented on slides by a Kodak Carousel projector. The images from the projector were monitored by a television camera and transmitted to the T.V. monitor. An electromagnetic shutter (Gerbrant) on the lens of the projector controlled the brief $20 \mathrm{msec}$. presentation of the stimulus. Advancement of the carousel and the shutter opening were controlled by the computer.
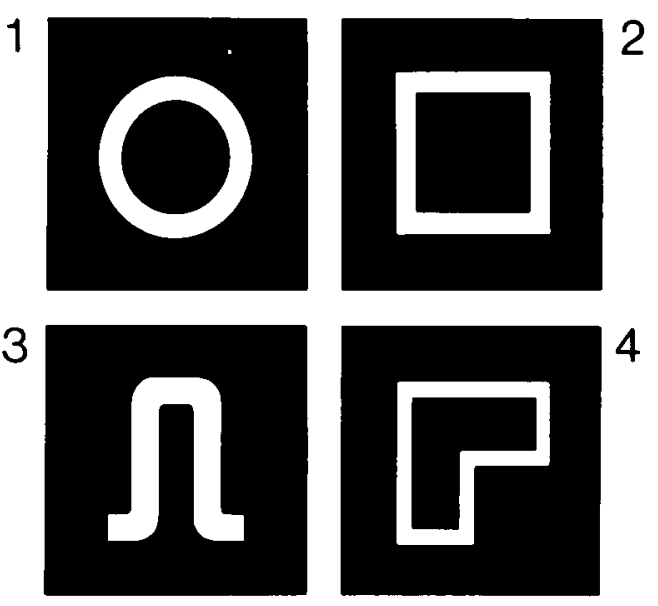

Figure 1-Stimulus shapes. 1. circle 2. square 3. omega 4. el

The size of the shapes on the T.V. screen was approximately $6 \mathrm{~cm}$. by 6 $\mathrm{cm}$.; therefore the shapes subtended an angle of $3.6^{\circ}$ at the centre of the subject's visual field. The luminance measured at the screen was $10 \mathrm{ft}$.candles for the background and approximately $25 \mathrm{ft}$-candles for all four stimuli.

The four stimuli were presented in a random sequence at from 3-5 sec. intervals until 30 trials of each shape were collected. A total of 25 runs of 120 trials were gathered for the 12 subjects.

The electrodes were Grass gold discs applied with Grass electrode paste according to the International 10-20 system at the standard locations of $\mathrm{O}_{2}$, $0_{1}, P_{4}, P_{3}, C_{4}, C_{3}$, and $\mathrm{CZ}$. All recording electrodes were referred to the contralateral ear or to the left ear for midline locations.

The EEG was recorded on a Beckman Dynograph Type $R$ with an effective system band width of $0.5-30$ $\mathrm{Hz}$ (3dB points). From the Dynograph, the EEG signals on seven channels were led to A-D converters of the computer and sampled every $2 \mathrm{msec}$. for a $512 \mathrm{msec}$. epoch, beginning $56 \mathrm{msec}$. before the stimulus presentation. Single trials ( 256 points per trial) were stored on the disc of the computer system 
(PDP 11/20) and all data was transferred to Dectapes for permanent storage at the end of each paradigm.

The experiments were all controlled in real time by a program which ran on the PDP 11/20 system. An automatic artefact rejection routine was included. This routine checked each trial sample to determine if it exceeded some preset amplitude limits, and if it did the trial was not saved. The program then continued the experiment until 30 trials of each shape had been collected.

Thirty trials for each stimulus shape were averaged to obtain the VERs for examination. With subjects that were tested more than once, the responses were found to be nearly identical from one experiment to another. The responses showed some variation in latency and configuration between subjects, but did exhibit essentially the same series of components for all subjects.

The peaks in the waveforms were labelled as follows: the first major positive peak seen in all subjects at about $95 \mathrm{msec}$. was called $\overline{\mathrm{P} 95}$. Following $\overline{\text { P95 }}$ the next large negative peak was called $\overline{\mathrm{N}} 150$ and the broader positive wave following it was called

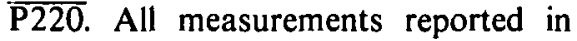
this paper were made on occipital recordings because the components were most clearly defined at these locations in all of the subjects.

The $\lambda$ ratios (used by John et al., 1967) were computed from averages of 10 trials from the formula $\lambda=\left(\mathrm{d}_{1,3}+\right.$ $\left.d_{2,4}\right) /\left(d_{1,2}+d_{3,4}\right)$, where $d_{i j}$ is the absolute value of the root mean square (r.m.s.) difference between waveforms $i$ and $\mathrm{j}$ (r.m.s. $=\mathrm{xi}^{2} / \mathrm{n}$ where $\mathrm{si}$ is the ith sample of the waveform with the value of the baseline as computed from the first 3 points $(18 \mathrm{msec}$.) of the response subtracted from it). The subscripts 1 and 2 denote the two replicated responses to one shape and 3 and 4 denote the replicated responses to the second shape. These ratios were computed for the five subjects whose data had been transferred to the IBM 370 computer system for discriminant analysis as reported below.

1. The discriminant analysis program used in this study and all others referred to in this paper is the Stepwise Discriminant Analysis Program (SWDA) available as part of the UCLA BMD program package (Dixon 1965, Program 07M).
For discriminant analysis (SWDA) ${ }^{1}$ the digitized trials were transferred on tape to files on the University IBM 370 system. During the transfer, the digitized trial representation was reduced from 256 points to 67 points by omitting the points representing the first $50 \mathrm{msec}$. of the pre-stimulus baseline, then taking the average of each of 67 sets of 3 points that followed, so that each value was the average voltage of a $6 \mathrm{msec}$. interval.

The BMD:07M program was run for either 2 or 4 groups (each group consisting of 30 trials elicited by a different shape). The number of variables per observation (i.e. single trial) was 67 as
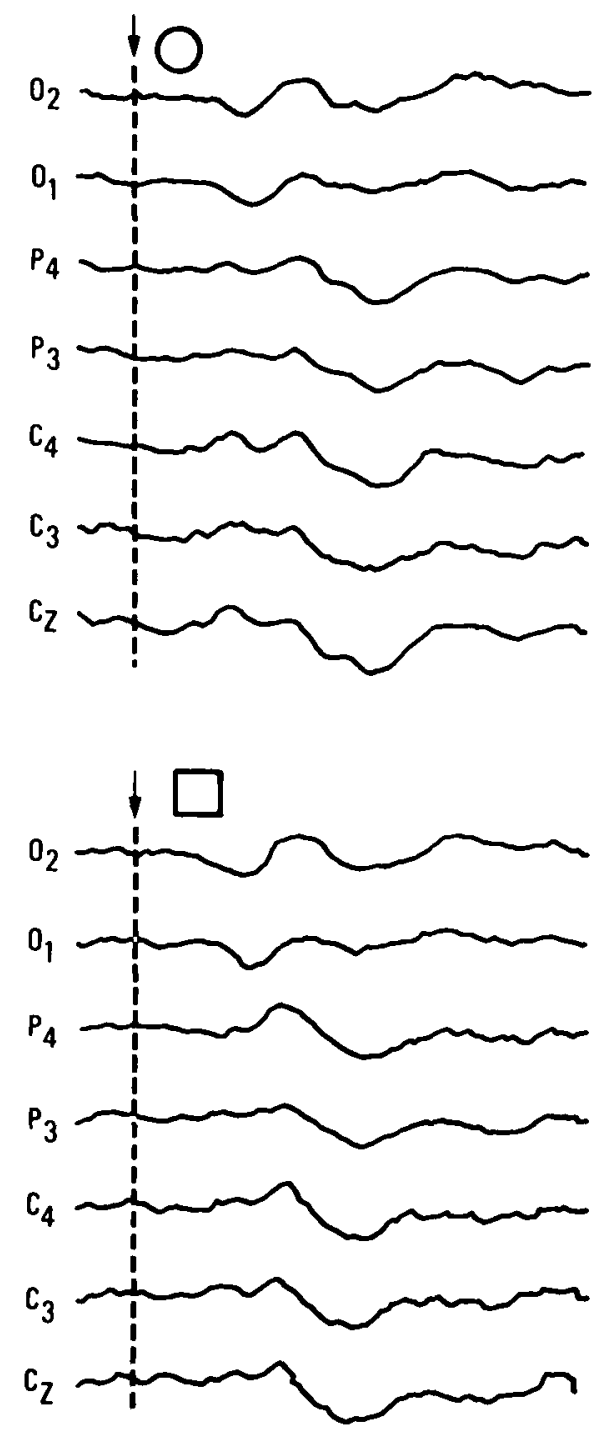

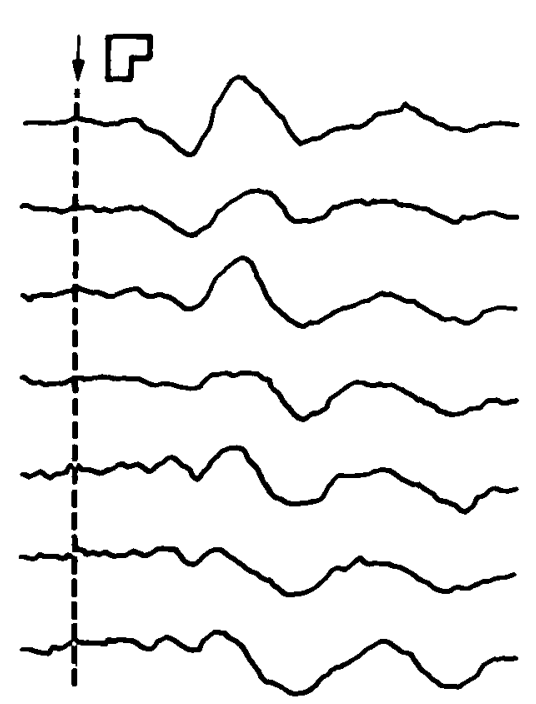

indicated in the previous paragraph and the program selected 6 or 7 of these (i.e. 6 or 7 latency points) as the basis of the discriminant function (DF). Pilot studies showed that allowing the program to run through more than 7 steps (i.e. to choose more variables than this) did not improve the discrimination and so the program was instructed to stop at this number of steps.

For testing one of these DFs, data from all of the experiments for the same subjects for the same channel that had not been used for input to the BMD:07M program that determined the DF, were put in a single file. These new trials were then each classified by

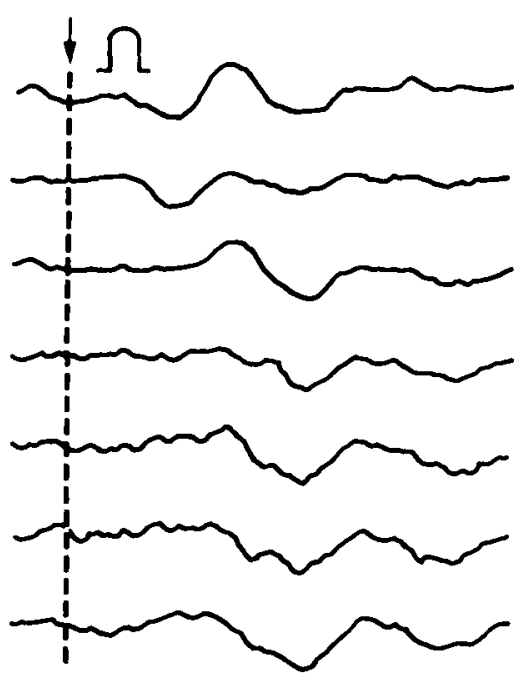


TABLE I:

$\begin{array}{llll} & P_{1} & N_{2} & P_{2} \\ \text { square } & 2.9 \pm 2.4 & 6.4 \pm 4.0 & 6.2 \pm 3.1 \\ \text { circle } & 3.4 \pm 2.4 & 5.2 \pm 2.8 & 7.2 \pm 4.5 \\ \text { omega } & 3.8 \pm 3.1 & 8.1 \pm 3.8 & 10.5 \pm 5.5 \\ \text { el } & 4.8 \pm 3.4 & 10.4 \pm 4.8 & 9.5 \pm 5.0\end{array}$

Amplitudes of the three principal components of the responses at $\mathrm{O}_{2}$ to the four shapes presented in paradigm 1. Each value (in $\mu \mathrm{v}$ ) is the mean and standard deviation for 12 subjects.

TABLE II:

Square-el comparison Circle-omega comparison

\begin{tabular}{lllll}
\hline location & $0_{2}$ & $0_{1}$ & $0_{2}$ & $0_{1}$ \\
\hline Subject & & & & \\
SP & 2.4 & 5.4 & 1.7 & 2.2 \\
ML & 2.8 & 3.5 & 2.4 & 1.0 \\
MP & 3.5 & 1.8 & 2.6 & 1.8 \\
SC & 1.7 & 1.5 & 1.0 & 1.2 \\
MDL & 3.6 & 1.2 & 2.1 & 1.9 \\
\hline 5 subject & 2.8 & 2.7 & 1.9 & 1.6 \\
mean & & & & \\
\hline
\end{tabular}

Mean $\lambda$ values for each subject. Each value in the Table is the mean $\lambda$ for 4 pairs of averages of 10 trials each of the 50 to $350 \mathrm{msec}$. interval following the stimulus.

\section{TABLE III:}

$\begin{array}{lll}\text { Subject } & 0_{2} \text { (right) } & 0_{1} \text { (left) } \\ \text { MP }(260) & 41 \% & 44 \% \\ \text { SC }(480) & 37 \% & 37 \% \\ \text { VS }(240) & 39 \% & 40 \% \\ \text { ML }(360) & 35 \% & 41 \% \\ \text { mean of } 4 \text { subjects } & 38 \% & 40 \%\end{array}$

Performance of the "Four shape" Discriminant Functions. Percentage of trials correctly classified by the BMD:07M Stepwise Discriminant Analysis Technique on data from right and left occipital locations. Total number of trials classified for each subject is given in parentheses. All percentages given are significantly* greater than the $25 \%$ level expected with a random classification $(\mathrm{p}=.01)$. For $35 \%$ $\mathrm{z}=3.58$ which is greater than $\mathrm{z}_{01}=2.57$.

- significance determined by evaluating the test statistic $z=\hat{p}-p / \sqrt{p q / n}$ where $\hat{p}$ is the observed proportion, $\mathrm{p}$ is the expected proportion, $\mathrm{q}=1-\mathrm{p}$ and $\mathrm{n}$ is the sample size. $\mathrm{z}$ is considered to be normally distributed.

the DF and the results of this testing were expressed as percentages of correctly classified trials.

\section{RESULTS}

Consistent differences between the evoked responses to some of the four different shapes were observed. Fig. 2 shows the responses to the four shapes at the seven recording sites for one subject. In order to define and quantify these intershape differences in the occipital regions, the three techniques of visual inspection with measurement of peak amplitude and latency, computation of a $\lambda$ ratio, and SWDA were used.

\section{Peak Latency and Amplitude \\ Measurements.}

There were no significant differences in the latency of the $\overline{\mathrm{P} 95}, \overline{\mathrm{N}} 1 \overline{50}$ or $\overline{\text { P220 }}$ components between the different shapes. The actual peak latency values for these components in the 12 subjects were $95 \pm 21 \mathrm{msec}$., $148 \pm 23 \mathrm{msec}$., $218 \pm 23 \mathrm{msec}$. respectively. There were also no significant differences in the amplitude or latency of any of the components between the response from the right and left occipital electrodes.

There were, however, significant differences in the amplitudes of two of these components when certain pairs of shapes were compared. The mean amplitude of each of the three components recorded at $\mathrm{O}_{2}$ for the four shapes for 12 of the subjects is shown in Table I. The amplitudes of the $\overline{\mathrm{N} 150}$ component of the square vs. el, circle vs. omega and circle vs. el; and of the

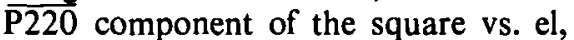
circle vs. omega and square vs. omega were all found to be significantly different using Scheffe's (1959) method of testing multiple contrasts $(p=.01)$. There were no significant differences ( $p$ $=.01$ ) in the amplitude of the P95 component. It is noted that Scheffe's method is based on a two way analysis of variance and thus it is essentially testing the difference between the shapes for each of the 12 subjects.

\section{Results of the $\lambda$ Descriptor Computations.}

$\lambda$ values for pairs of different shapes were computed for each subject as described in the Methods section. Table II presents the mean value of this $\lambda$ statistic for the five subjects for the square-el and circle-omega pairs at $0_{1}$ and $\mathrm{O}_{2}$. Each value in this Table is the mean of four values computed for that subject, location, and pair of shapes. All four averages used to compute one $\lambda$ value were taken from data obtained in a single experimental session. All values in the Table are based on the 50 - $350 \mathrm{msec}$. post-stimulus interval of the averages. The mean value of $\lambda$ for the square-el comparison was 2.7 and for the circle-omega pair was 1.7 for the five subjects. The fact that the mean value of this ratio is greater than one indicates that the evoked potential waveforms of the square and el and of the circle and omega are different and this statistic provides a quantitative index of the differences. The $\lambda$ values were also computed for the $0-350$ msec. and $120-300 \mathrm{msec}$. intervals. The values for the other intervals were not very different from the ones given. The mean of all values for the largest 0 - 350 msec. interval was 2.1 , for the 50 - $350 \mathrm{msec}$. interval reported in Table II 2.3 , and for a smaller $120-300 \mathrm{msec}$ interval it was 2.2 .

\section{Results of the Discriminant}

Analysis Technique.

The work with the BMD:07M and the testing of the functions produced by it was done in two phases. In the first phase, data collected from four subjects were used. Single trials from all four stimulus shapes were submitted to the BMD:07M program at once, hence the resulting DFs were intended to classify any single trial (or observation) as belonging to one of the four possible groups (shapes). In the second phase, 
the data from only two stimulus shapes at a time were submitted to the program.

In Table III the results of testing the DFs classifying all four shapes for the four subjects on data from right and left occipital locations are presented. The percentages given in the Table are the proportions of the total number of trials the DF correctly classified. The classifications made on the trials used to compute the DF (i.e. a posteriori classification) were included in the percentages and total numbers of trials given in this Table.

One hundred twenty trials, 30 of each shape, were used to compute each DF. It can be seen from the Table that the DFs classified from 35 to $44 \%$ of the waveforms from the single trials correctly, proportions which are significantly greater than the level of $25 \%$ expected with a random classification $(z=3.58$ for $35 \%$ which is greater than $\mathrm{z} .01^{2}=2.57$ ).

In the second phase of the application of discriminant analysis to the evoked potential data, DFs for only two shapes at a time were computed and tested. All six possible pairs of the shapes for one subject were tested and the results are presented in Table IV. It was found that the DFs for the squareel and circle-omega pairs always had the lowest $U$ values ${ }^{\prime}$ of any of the six pairs, and also had the highest percentage of correct classifications of new data.

These two pairs of shapes were then tested for another four of the subjects. For each subject 30 responses from each shape were used to compute the DF and then the DFs success in classifying other data correctly for that subject was evaluated.

The results of the two-group DFs computed and tested on these five subjects are presented in Table V. In this Table the percentage classifications made $a$ posteriori and on new data are presented separately. Only one of the twenty functions (it is marked by an asterisk) did not perform significantly better than the chance $50 \%$ level in

1. U statistic provided in BMD:07M to evaluate the statistical significance of the separation of the two input groups.

TABLE IV:

$\begin{array}{lll} & & \text { NEW DATA } \\ \text { Square vs. omega } & 88 & 67 \%(290) \\ \text { Square vs. circle } & 70 & 59 \%(290) \\ \text { Circle vs. el } & 82 & 66 \%(230) \\ \text { Square vs. el } & 87 & 76 \%(320) \\ \text { Circle vs. omega } & 80 & 71 \%(380)\end{array}$

Performance of the "two-shape" discriminant functions for five of the six possible pairs of shapes for one subject (MP-data from $0_{1}$ ) Number of correct predictions made of single trials on the data used to make the function and on new data. Number of new trials classified given in brackets. All percentages given of correct classifications are significantly higher than $50 \%(\mathrm{p}=.01)$.

\section{TABLE V:}

Square-el functions:

\begin{tabular}{lllll} 
& \multicolumn{2}{l}{$0_{2}$ Data } & \multicolumn{2}{c}{$0_{1}$ Data } \\
Subject & a posteriori & new data & a posteriori & new data \\
MP & $75 \%$ & $65 \%(240)$ & $87 \%$ & $76 \%(320)$ \\
SC & $72 \%$ & $63 \%(240)$ & $72 \%$ & $65 \%(240)$ \\
ML & $83 \%$ & $62 \%(240)$ & $75 \%$ & $70 \%(440)$ \\
MD & $87 \%$ & $71 \%(190)$ & $87 \%$ & $62 \%(190)$ \\
SP & $83 \%$ & $58 \%(140)^{*}$ & $93 \%$ & $68 \%(140)$ \\
\hline mean of subjects: & $80 \%$ & $64 \%$ & $83 \%$ & 68 \\
\hline Circle-omega functions: & & & & \\
MP & $80 \%$ & $61 \%(380)$ & $80 \%$ & $71 \%(380)$ \\
SC & $77 \%$ & $65 \%(190)$ & $77 \%$ & $63 \%(190)$ \\
ML & $82 \%$ & $65 \%(320)$ & $82 \%$ & $60 \%(320)$ \\
MD & $93 \%$ & $65 \%(220)$ & $78 \%$ & $60 \%(220)$ \\
SP & $70 \%$ & $59 \%(140)$ & $72 \%$ & $61 \%(140)$ \\
\hline mean of subjects: & $80 \%$ & $63 \%$ & $78 \%$ & $63 \%$ \\
\hline
\end{tabular}

Performance of the "two-shape" discriminant functions. Number of correct predictions made of single trials on the data used to make the function (a posteriori) and on new data. Number of new trials classified given in brackets. All functions were made with 60 trials. All percentages given of correct classifications are significantly higher than $50 \%(\mathrm{p}=.05)$ except the one marked $*$. Significance determined by the method described in Table IV footnote. $z=2.13$ for $59 \%(n=140)$ which is greater than $z .05=1.96$

classifying new data from the same subject. The classification of the square and el shapes was slightly better than for the circle-omega pair, but the difference was not statistically significant. There was no significant difference between the performance of DFs from data from the right or left hemisphere.

As described in the Methods section, the BMD:07M essentially chooses a subset of variables (latency points) on which to base the DF. The distribution of these latency points chosen for the fourteen DFs for data from $\mathrm{O}_{2}$ whose performance is described in Tables III and $\mathrm{V}$ is shown in Fig. 3. The solid circles show points chosen in the first or second step of the computation of the DF and the outlined circles show points chosen in the remaining four steps. There was a tendency for the points chosen in the first two steps of the SWDA to be clustered around the $150 \mathrm{msec}$. and $220 \mathrm{msec}$. regions of the responses, which corresponded to the latency of the $\overline{\mathrm{N} 150}$ and $\overline{\mathrm{P} 220}$ components noted in the visual assessment of the configuration of the responses.

\section{DISCUSSION}

The main purpose of this study was to demonstrate and quantify the differences in visual evoked responses to different geometric forms. Evoked response differences were quantified by a variety of analysis techniques including (1) measuring the amplitudes of selected components, (2) computation of a descriptive ratio called $\lambda$ by John et al. (1967) in a study similar to part of this one, and (3) performance of classification functions computed by Stepwise Discriminant Analysis on new data. A comparison of the results of these three techniques shows them essentially to be in agreement with each other.

The technique of testing the SWDA functions on new data (that was not 
used to create the function) avoids relying on statistics such as the $U$ value produced by the program to measure the validity of the intergroup differences, and hence reduces the importance of satisfying the theoretical assumptions of linear independence of the variables and Gaussianity of the interobservation variance. It uses single trial data rather than averages and thus utilizes the variance of the single trials included in the averages, which is ignored in other techniques and which is important in determining the statistical significance of the findings.

From Fig. 3 it can be seen that very few of the first two points chosen by the DFs were found before $120 \mathrm{msec}$. The figure also indicates some tendency for the latency points chosen for the discriminant functions (particularly those chosen in the first two steps) to be clustered around the 150 and $220 \mathrm{msec}$. regions of the waveforms. These are the latency values of the components $\overline{\mathrm{N} 150}$ and $\overline{\mathrm{P} 220}$ that were found to differ significantly in amplitude between the pairs of shapes.

The $\lambda$ ratio statistic was computed to enable a comparison to be made between the results of this study and the one of John et al. (1967). These authors suggest that a $\lambda$ value of greater than 1 indicates the waveforms are significantly different. A value greater than 1 does indicate that the difference between the waveforms from two different stimuli is greater than the difference between the waveforms from two replications of the same stimulus. However, it is not possible to determine at what value this ratio would achieve statistical significance, nor does it indicate at what latency points the differences occur. The mean $\lambda$ values determined for the data of the present study for the square and el or circle and omega comparisons (see Table II) are similar to those reported for all of the intershape comparisons in John's study. The $\lambda$ values computed in this study for the square and circle comparison, however, were not found to be consistently greater than 1 (and consequently are not reported in the Table). John et al. did not separate their results for the different shape comparisons so the exact significance of this discrepancy is uncertain, but it suggests

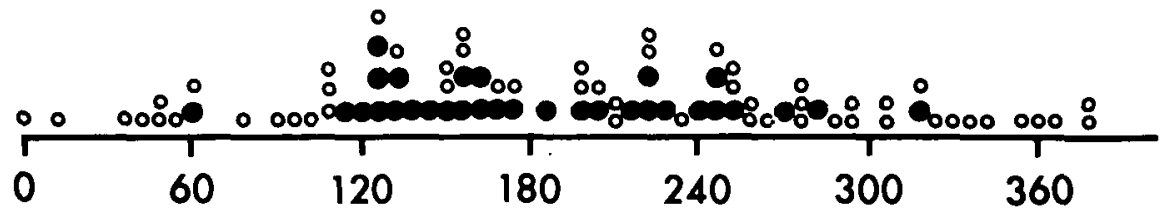

msec After Stimulus

Figure 3-Distribution of the variables chosen for the DFs for both the four group and two group discriminations of data from $\mathrm{O}_{2}$. Each dot shows the latency of one variable. The solid dots indicate a variable chosen in the first or second step of the SWDA program and the outlined dots indicate variables chosen in the subsequent 4 steps of the program.

that the VER differences between square and circle responses were much smaller in the present study than in this previous one.

Thus it has been confirmed that the occipitally recorded VER is significantly different for some patterned visual stimuli that differ in symbolic meaning, suggesting that perceptual processes may be reflected in the VER. However, because the stimuli also differ slightly in their physical characteristics it is possible that these are a significant factor in determining the observed waveform differences.

The presentation of all four shapes in random order in a single run insured that general correlates of the subject's state such as arousal and attention, which are known to affect the response configuration, were the same for all of the stimuli. The contrast level, focus, and size of the stimuli were always equal for the four shapes, and they all had very nearly equal lengths of contrasting borders and luminance on projection. Thus, although various authors have reported changes in the evoked response configuration due to each one of these factors (Rietveld et al., 1967; Regan, 1972), none of them could account for the differences demonstrated in this study.

Some authors (Regan, 1972; MacKay, 1969) have suggested that the presence of corners in the stimulus pattern is an important determinant of evoked response configuration, but definite evidence for this is not yet available and our study does not provide any. The demonstration of Rietveld et al. (1967) that checkerboards produce larger responses than stripes and that blurring the corners of the checkerboard changed the evoked responses is cited as support for the suggestion of Regan and MacKay, but
Rietveld's findings could be explained on the basis of changes in total contrasting border length. Furthermore, our study has shown that the evoked responses to circle and square were quite similar, although the stimuli differed by four corners, whereas the omega and circle differed by only two corners and produced quite different responses.

Harter's (1970) findings on the effect of retinal eccentricity and check size may be quite relevant and could at least partially explain why these patterns may evoke different responses. He showed that with a 90' check there was about a $3 \mu \mathrm{v}$ drop in amplitude of the 180 msec. positive component when the area stimulated was changed from the central 0 to $1.5^{\circ}$ to the more peripheral $1.5^{\circ}$ to $4.0^{\circ}$ of the visual field. In the present experiments, both the el and the omega had more contrasting borders in the central $1.5^{\circ}$ of the subject's visual field than the circle or square did.

Campbell's laboratory has provided some evidence that steady-state evoked potentials reflect the spatial frequency of the visual stimulus (Blakemore and Campbell, 1969; Campbell and Robson, 1968) and a recent paper by Musso and Harter (1975), recording transient evoked potentials, confirms the findings. These latter authors found a masking effect on the $110 \mathrm{msec}$. negative component of the evoked responses to the second stimulus of a pair only if the first stimulus contained the same spatial frequencies as the second. It may be that the greater number of spatial frequencies contained in the el and omega compared to the square and circle is an important determinant of the higher amplitude of the earlier parts (around $150 \mathrm{msec}$. 
latency) of the responses to the unfamiliar shapes that were seen in this study, and some further work is in progress to clarify this possibility.

All of these studies suggest that physical properties of the stimulus are important in determining VER configuration only up to $180 \mathrm{msec}$. after the stimulus. Since we have shown VER differences in a later latency period than this (in a paradigm where more non-specific factors related to attention levels or significance that are known to affect later components did not change between stimuli), it seems likely that these later differences are related to more complex features of the stimuli, such as symbolic meaning.

In conclusion, it has been shown in this experimental work that the occipital VER is different for some different geometric patterned stimuli. These differences were confirmed using a variety of quantitative techniques, including SWDA. It is believed that some as yet incompletely defined physical characteristics of the stimuli can account for the early latency waveform differences. The critical physical characteristics are probably the contour density in the central $1-2^{0}$ (foveal) of the visual field and/or the spatial frequencies contained in the pattern. Differences in later parts of the waveform (after approximately $180 \mathrm{msec}$. latency) may result from other cerebral processes related to the determination of the symbolic meaning of the patterns.

\section{ACKNOWLEDGEMENTS}

We thank Jan Galloway and Michael Baker for technical assistance and Louise Stevenson for manuscript preparation.

\section{REFERENCES}

BLAKEMORE, $C$. and CAMPBELL, F. W. (1969) On the existence of neurons in the human visual system selectively sensitıve to the orientation and size of retinal images. J. Physiol. 203: 237-260.

BUCHSBAUM, M. and FEDIO, P. (1969) Visual information and evoked responses from the left and right hemispheres. Electroenceph. Clin. Neurophysiol. 26: 266272.

CAMPBELL, F. W. and ROBSON, J. G. (1968) Application of Fourier analysis to the visibility of gratings. J. Physiol. 197: 551566.

DIXON, W. J. (1965) (Ed.) BMD - Biomedical Computer Programs, Univ. of California, Los Angeles.

DONCHIN, E. (1969) Data analysis techniques in average evoked potential research. In Averaged Evoked Potentials. (eds.) E. Donchin \& D. B. Lindsley. NASA, Sp-191, Washington, D.C. p 199-236.
HARTER, M. R. (1970) Evoked cortical responses to checkerboard patterns: effect of check-size as a function of retinal eccentricity. Vision Res., 10: 1365-1376.

HARTER, M. R., and WHITE, C. T. (1968) Effects of contour sharpness and check size on visually evoked cortical potentials. Vision Res., 8: 701-711.

JOHN, E. R. and HERRINGTON, R. N. and SUTTON, S. (1967) Effects of visual form on the evoked response. Science 155: 14701471.

MACKAY, D. M. (1969) Evoked brain potentials as indicators of sensory information processing. Neurosciences Res. Prog. Bull 7, No. 3: p 181-273.

MUSSO, M. F. and HARTER, M. R. (1975) Visually evoked potentials and selective masking with patterned flashes of different spatial frequencies. Vision Res., 15: 231. 238.

REGAN, D. (1972) Evoked Potentials in Psychology, Sensory Physiology and Clinical Medicine. Chapman and Hall (London).

REM, M. A. and SCHWARTZ, M. (1976) Retinal versus central processes in determin. ing averaged evoked response waveforms. Physiol. Behav. 16: 705-709.

RIETVELD, W. J., TORDOIR, W. E., HAGENOUW, J. R. B., LUBBERS, J. A. and SPOOR, T. A. C. (1967) Visual evoked responses to blank and to checkerboard patterned flashes. Acta Physiol. Pharmacol. Neerl., 14: 259-285.

SCHEFFE, H. (1959) Analysis of Variance., John Wiley \& Sons.

SPEKREIJSE, H., VAN DER TWEEL, L. H., and ZUIDEMA, T. (1973) Contrast evoked response in man. Vision Res. 13: 1577-1601. 